\title{
Extracellular Potential Recording of Patterned Rat Olfactory Bulb Neuronal Network Using Planar Microelectrode Arrays
}

\author{
Jian TIAN \\ Department of Biomedical Engineering \\ Zhejiang University \\ Hangzhou, China \\ e-mail: tianjian@zju.edu.cn \\ Bobo HUANG \\ Department of Biomedical Engineering \\ Zhejiang University \\ Hangzhou, China \\ e-mail: 15158073581@zju.edu.cn
}

\author{
Chunlong TU \\ Department of Biomedical Engineering \\ Zhejiang University \\ Hangzhou, China \\ e-mail: tcl@zju.edu.cn \\ Xuesong YE* \\ Department of Biomedical Engineering \\ Zhejiang University \\ Hangzhou, China \\ e-mail: yexuesong@zju.edu.cn
}

\begin{abstract}
The olfactory bulb is an important structure in olfactory information processing. Exploring electrophysiological characteristics of dissected olfactory bulb neuron in vitro is helpful to improving the understanding of the mechanism of the olfactory information processing. In this work, a novel neural chip was developed based on the combination of the microelectrode array and patterned microstructures with the advantages of controllable growth of neurons and long time potential recording. Topographical patterning was used to define position of neuron soma and to guide the neurite outgrowth. A cell culture device with micro wells and micro trenches was fabricated in polydimethylsiloxane using soft lithography. The olfactory bulb neurons were cultured on the cell culture device. At day 8 of the in vitro culture, the device with patterned neuronal network was put upside down on the top surface of a commercially available microelectrode array chip. The micro wells were aligned letting neurons sit on the electrodes. Spike signals of neurons were recorded when the neuronal network was subjected to $1 \mathrm{mM}$ glutamate stimuli. This convenient method by which the olfactory bulb neural chip was fabricated is feasible for extracellular potential recording of patterned neurons, and may be useful in the further studies of olfactory information processing.
\end{abstract}

Keywords-olfactory bulb neuron; patterned neuronal network; polydimethylsiloxane; microelectrode arrays

\section{INTRODUCTION}

Odor information can be coded by temporal patterns of activity in the olfactory bulb (OB) [1]. The axonal and dendritic processes have irregular orientations in the glomerular layer of the OB. This renders analysis of extracellular potentials from OB to be difficult. As a result, our understanding of the neuronal properties and synaptic actions lags behind our understanding of synaptic connections [2]. Exploring the electrophysiological properties for OB cells is a critical first step to determine the local circuit connections and how they participate in sensory processing. Additional studies examining synaptic transmission in larger populations of olfactory bulb neurons will be essential to determine the relative contributions of temporal and spatial coding in the mammalian olfactory system.

As the mechanisms of odor discrimination and olfactory information encoding remain unclear, some electrophysiological recording techniques such as the implanted electrodes [3] and patch-clamp [4], have been employed to investigate the odor information processing. The patch-clamp offers direct information on olfactory transduction in single cell, but it is not available for longterm recording and the throughput is low. Besides, most of the studies on $\mathrm{OB}$ were accomplished in vivo, where the experimantal results might be influenced by endogenous sources of correlation [5] such as variable levels of arousal. The electrical recording of neurons in vitro is a helpful approach to investigate the properties of the OB cells. The extracellular measurement using a microelectrode array (MEA) has been proved an effective long-term recording technique for neural cells in vitro, which is capable of simultaneously measurements of multiple cells.

The MEA-based olfactory neural chip has been reported for the researches in olfactory transduction [6]. These existing olfactory-chips were valuable for olfactory information coding researches. However, the connections between neurons cultured on these neural chip were random and complicated. The cells often escaped from the electrodes in a long-term cultivation. Changes in the relative position of the cells and recording sites were unpredictable, resulting in a bad signal to noise ratio. Besides, an electrode on the MEA often receives the signals from multiple neurons. These uncertainties led to difficulties in analyzing the network structure and building a mathematical model of the neuronal network. An MEA system with controlled cell positions and connections may be a possible solution to these problems. Unfortunately, to the best of our knowledge, the patterned OB neural chip used for the research of the olfactory system has not been reported so far. 
Cell patterning is a popular technique for defining cell positions in many applications. Local cellular environment can be controlled at the micron scale by this approach, which is particularly useful in single-cell-based researches [7]. Techniques with chemically patterned growth substrates [8] or three-dimensional microstructures [9] were used successfully for cell patterning. Bani-Yaghoub et al.[10] made specific topographic structures on polydimethylsiloxane (PDMS), and modified the property of PDMS surface with fluoroalkylsilane derivative solution to obtain a patterned neuronal network. If neurons are confined to the top of the microelectrodes merely by threedimensional microstructures, placing the neurons into the microstructure seems to be cumbersome, and the neurons may be damaged during the mechanical transferring. For the chemically patterning, the neurites may escape from the small patterned micro channels during long time culture. In present work, the rat OB neuronal networks were cultured on the neural chip with the capability of controllable growth of neurons. A simple method combing three-dimensional microstructures and chemical modification was developed to form an OB neuronal network on the MEA surface, where a PDMS device with micro wells and micro trenches was used for OB neuron culture. The PDMS surface was treated with air plasma and fluoroalkylsilane to make it have both celladhesive region (micro wells and micro trenches) and cellrepellent region (top surface of the device). The neurons survived more than a week in the micro wells. After the neuronal network was formed in the cell culture device, the device was placed upside down on the surface of the MEA, the spike signals of $\mathrm{OB}$ neurons were recorded after the administration of glutamate. This convenient method by which OB neural chip was fabricated is feasible for extracellular potential recording of patterned OB neurons, and may be useful in the further studies of olfactory information processing.

\section{MATERIALS AND METHODS}

\section{A. Device Fabrication}

A master mould was made in SU-8 photoresist on a silicon wafer by lithography techniques. A photomask with a micro pattern was produced. The photoresist SU-8 was dispensed onto a silicon wafer. By a spin coater, the wafer was coated with the photoresist of $40 \mu \mathrm{m}$ thick. Next, the coated wafer was baked on the hotplate. After cooled to room temperature, the photoresist layer was covered by the photomask and exposed to UV light. Then, the wafer was baked and developed with SU-8 developer. By this way, the master mould with square pillars and rectangular ridges was fabricated. PDMS pre-polymer was poured onto the mould and spin-coated to a thickness greater than $1 \mathrm{~mm}$. After cured, the PDMS was peeled off from the mould. The cured PDMS was cut into square blocks of about $2 \mathrm{~mm}$ thick. In the center of the block surface, there was a $5 \times 5$ micro well array, the length of each micro well was $50 \mu \mathrm{m}$. Many $150 \mu \mathrm{m} \times 10 \mu \mathrm{m}$ rectangular micro trenches linked neighboring micro wells (Fig. 1). Both of the micro wells and micro trenches had the depth of $40 \mu \mathrm{m}$. Each side of the micro well array was connected with a $1 \mathrm{~mm} \times 5 \mathrm{~mm}$ rectangular cavity. The PDMS block was used as a cell culture device.

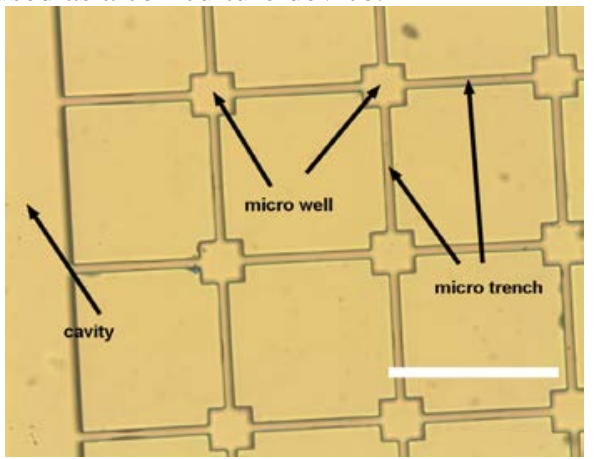

Figure 1. The PDMS cell culture device with micro wells $(50 \times 50 \mu \mathrm{m})$, micro trenches $(150 \times 10 \mu \mathrm{m})$ and cavities $(5 \times 1 \mathrm{~mm})$. Scale bar is $100 \mu \mathrm{m}$.

\section{B. Surface Preparation}

The PDMS culture device was piranha etched, rinsed in DI water, and blown dry to get rid of the toxins. The solution of fluoroalkylsilane [1:50 (heptadecafluoro-1, 1, 2, 2-tetrahydrodecyl) triethoxysilane/ethanol] was dropped on a flat PDMS film. 1 hour later, the PDMS film dried naturally. After ashed for 5 minutes at $10.15 \mathrm{~W}$ in a plasma cleaner, the PDMS culture device was placed on the PDMS film for 2 hours with the microstructured side attached to the film. Then, the cell culture device was removed. Poly-L-lysine (PLL) solution $(100 \mu \mathrm{g} / \mathrm{ml})$ was dropped onto the surface of micro wells and micro trenches in the culture device. The device with PLL solution was put into a vacuum drying oven to remove the air bubbles in the microstructures. The surface modification procedure is illustrated in Fig. 2. Contact angle measurement was employed to evaluate the surface modification efficient. The contact angles of the newly cured PDMS, the PDMS left in air for $2 \mathrm{~h}$ after being ashed and the PDMS coated with fluoroalkylsilane were measured.

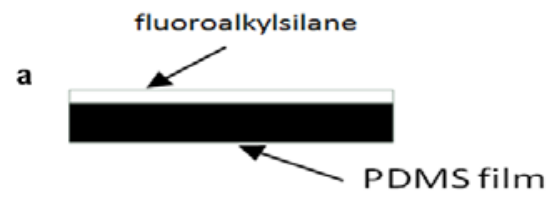

b

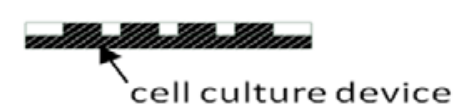

c

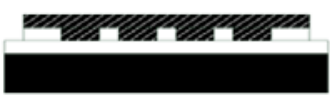

Figure 2. The surface modification procedure. (a) A cured PDMS film with fluoroalkylsilane on its top surface. (b) The PDMS cell culture device. (c) After being ashed, the cell culture device was placed on the top of a PDMS film with fluoroalkylsilane. This made the region except micro wells and micro trenches hydrophobic. 


\section{OB Neuronal Cell Culture}

OB neurons were obtained from P1 Sprague-Dawley rats (provided by laboratory animal center of Zhejiang province, Hangzhou, China). The procedures in this study were approved by the Zhejiang University Animal Care and Use Committee. The rats were decapitated and the OBs were dissected. The OBs were cut into small pieces and digested with $0.125 \%$ trypsin at $37^{\circ} \mathrm{C}$ for 10 minutes. Trypsinization was stopped using the culture medium consisting of DMEM and $10 \%$ fetal bovine serum. The cell solution was passed through a $74 \mu \mathrm{m}$ cell sieve and centrifuged at $1500 \mathrm{rpm}$ for 5 minutes. After removal of the supernatant, the cells were suspended in culture medium consisting of DMEM, high glucose, L-glutamine, $10 \%$ fetal bovine serum, and $1 \%$ penicillin-streptomycin. The cells were seeded on the PDMS culture device or a Petri dish at the concentration of $5 \times 10^{5}$ cells/ml. 24 hours later, excessive cells were rinsed away gently, the culture medium was replaced by the new medium which consisted of Neurobasal (Gibco), 1\% penicillinstreptomycin, $200 \mathrm{mg} / \mathrm{l} \mathrm{L}$-glutamine and 20 ml/l B27 (Gibco). The culture medium was replaced every three days. The temperature, humidity and $\mathrm{CO} 2$ concentration was kept $37^{\circ} \mathrm{C}$, $95 \%$ and $5 \%$, respectively, in the incubator.

\section{Extracellular Potential Recording}

On the 8th day of cell culture, the PDMS culture device was transferred to the culture chamber on a commercially available MEA chip (60MEA200/30-Ti, Multi Channel System). The culture device was submerged by the culture medium. The MEA chip had 59 round microelectrodes (diameter: $30 \mu \mathrm{m}$ ) and a reference electrode. The distance between adjacent electrodes was $200 \mu \mathrm{m}$, which conformed to the distance between the neighboring micro wells in the PDMS culture device. The micro wells were aligned to the electrodes under an inverted microscope. The open side of the micro well was attached to the MEA surface. The microelectrodes were seated in the center of the micro wells. Data were acquired with a sampling frequency of $50 \mathrm{kHz}$. The extracellular potentials of $\mathrm{OB}$ neurons in the microstructures were recorded for 100 seconds. After that, the culture medium was replaced with fresh Neurobasal medium containing $1 \mathrm{mM}$ glutamate. Signals were filtered between $300-2000 \mathrm{~Hz}$. Spikes were detected by a threshold set at a level three times higher than the standard deviation of the noise signal.

\section{RESULTS AND DISCUSSION}

\section{A. Results}

The hydrophilicity of PDMS surface modified by different procedures was evaluated by contact angle measurement (Fig. 3). The surfaces tested were the newly cured PDMS surface (group PDMS), the PDMS surface left in air for 2 hours after being ashed 5 minutes at $10.15 \mathrm{~W}$ (group PLASMA) and the PDMS surface coated with fluoroalkylsilane 2 hours after being ashed 5 minutes at 10.15W (the group labeled as "Fluoroalkylsilane modified cell culture device top surface"). The three groups had the contact angle of $117.26 \pm 0.54$ degree, $27.17 \pm 1.80$ degree and
$102.39 \pm 0.86$ degree (mean \pm SEM), respectively. When the PDMS culture device was placed on the PDMS film inked with fluoroalkylsilane solution, the surface except the inner wall of micro wells and micro trenches adsorbed the fluoroalkylsilane. Through the surface modification procedure described in the "Surface preparation" section , the micro wells and micro trenches in the PDMS cell culture device became hydrophilic, while the other area on the device was hydrophobic.
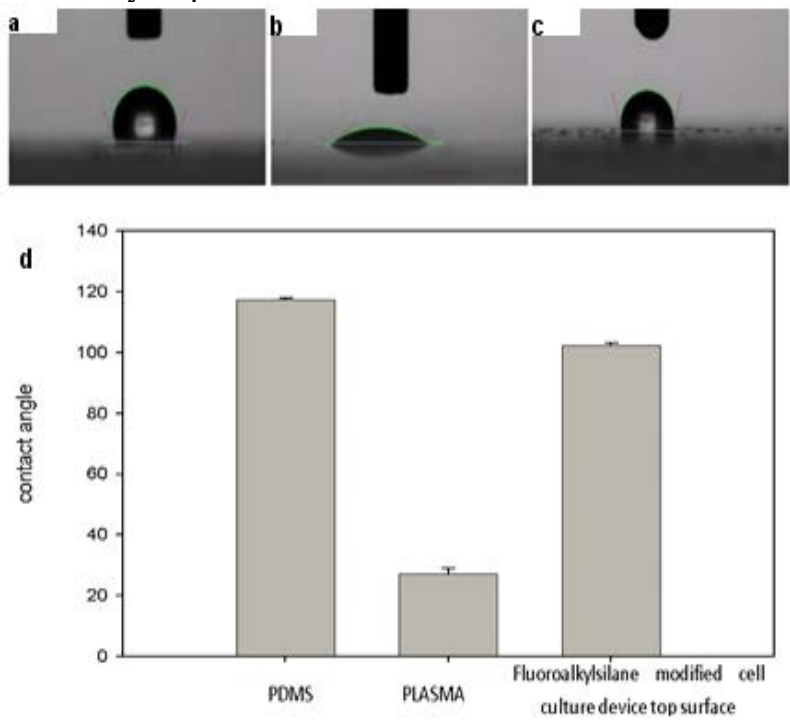

Figure 3. Contact angle measurement of three kinds of surface. (a) DI water dropped on newly cured PDMS. (b) DI water dropped on the PDMS left in the air for 2 hours after being ashed. (c) DI water dropped on the PDMS inked with fluoroalkylsilane (Fluoroalkylsilane modified cell culture device top surface) after being ashed. (d) The histogram of contact angles of three kinds of surface.

When the neuronal network formed, more than $70 \%$ of the micro wells were occupied by the OB cells. The use of serum-free culture medium prevented glia cells from packing the micro wells by reducing glia cell growth [11]. Axons and dendrites grew along the walls of the micro trenches. They reached the neurons in the adjacent micro wells (Fig. 4a) or stopped extending in the micro trenches where small cells were stranded (Fig. 4c,f). In some micro wells, more than one neuron was found (Fig. 4e). Most of the neurons grew into irregular shapes (Fig. 4b,d). This was rarely observed when neurons were cultured on a Petri dish. These neurons often clung to the corners of the micro wells. Fluorescence staining image revealed the connection between neurons in adjacent micro wells (Fig. 5). It was easy to confine the OB neurons to the micro wells. Extra cells on the cell-repellent surface of the PDMS culture device were effectively removed by gently rinsing the device. 


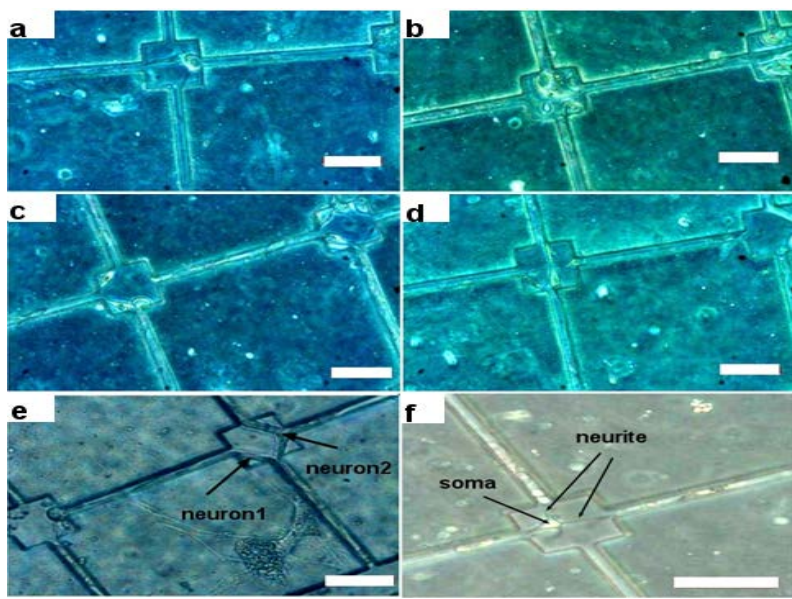

Figure 4. The OB neurons grew in the micro wells and micro trenches of the PDMS device. The scale bar is $50 \mu \mathrm{m}$.
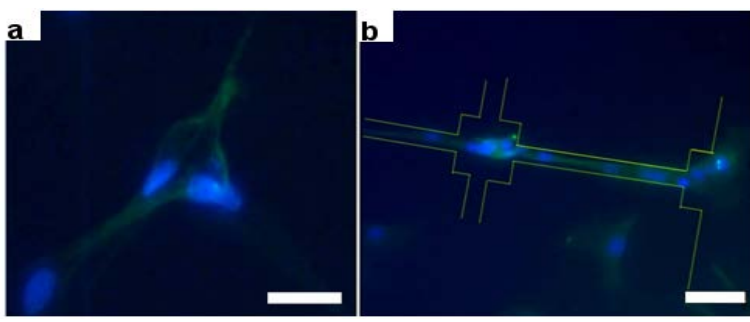

Figure 5. Fluorescence staining revealed the connection between neurons in adjacent micro wells, green: soma and neurites,blue: nucleus. The outline of micro wells and micro trenches was marked with yellow color. The scale bars is $50 \mu \mathrm{m}$.

After the PDMS device was placed upside down on the top surface of the MEA chip, the micro wells were aligned to the electrodes. The neurons faced to the electrodes (Fig. 6c).
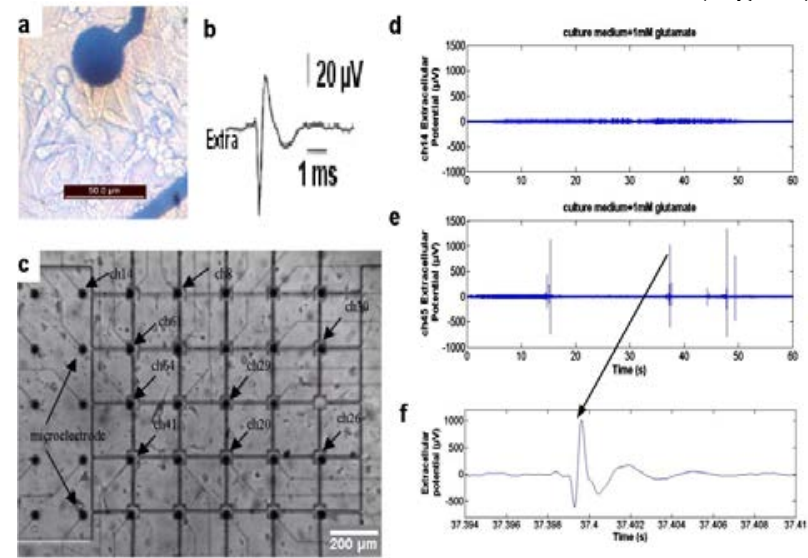

Figure 6. Extracellular potential recording of OB neurons. (a) The OB neurons were cultured directly on a MEA. Scale bar is $50 \mu \mathrm{m}$. (b) A picture adopted from Cohen and Mile's article [13]. It shows the extra potential of a neuron. (c) The PDMS cell culture device was placed on a MEA with the microelectrode aligned to the center of the micro well. Scale bar is $200 \mu \mathrm{m}$. (d) The signal recorded from the channel 14 marked in Fig. 6c. The channel 14 was silent as no cell located near the electrode. (e) The extracellular potential recorded from the channel 45 marked in Fig. 6c. (f) An enlarged picture of a spike signal in Fig. 6e. The signal is very similar with the extracellular potential in Fig $6 \mathrm{~b}$.
The signals recorded from eight channels before and after the administration of glutamate are shown in Fig. 7a and Fig. 7c. The extracellular potential signal recorded from channel 41 without glutamate stimuli is displayed in Fig. 7b. The noise was about $22 \mu \mathrm{V}$. After administration of $1 \mathrm{mM}$ glutamate, the neuron spikes appeared. The spike amplitudes were about $110-332 \mu \mathrm{V}$. The two phases of the spike correspond to the depolarization and repolarization of the neuron. According to another research, signals collected from a MEA had typical amplitudes in the range of 20$600 \mu \mathrm{V}$ and the thermal noise ranged from $10 \mu \mathrm{V}$ to $20 \mu \mathrm{V}$ [12]. Claverol-Tinture et al. [9] measured the extracellular potential of neurons with the open-well configuration. They found that some electrodes, although partially covered by the soma, remained electrically silent because the electrodes were grounded by the culture medium. Sealing the wells resulted in a better signal. In present work, the walls of micro wells and the MEA surface formed sealed compartments, preventing severe current leakage.

The spike signal recorded from the patterned OB neurons on the MEA (Fig. 6e,f) was compared with that reported by Cohen and Miles [13] (Fig. 6b). They had the similar shape and duration. Channel 14 was electrically silent (Fig. 6d) since no cell located near the electrode. In order to observe the propagation delay time of the signals, the spike waveforms marked in Fig. 7c are enlarged in Fig. 7d and Fig. 7e. The spike signals displayed in Fig. 7d have different shapes and propagation delay times.

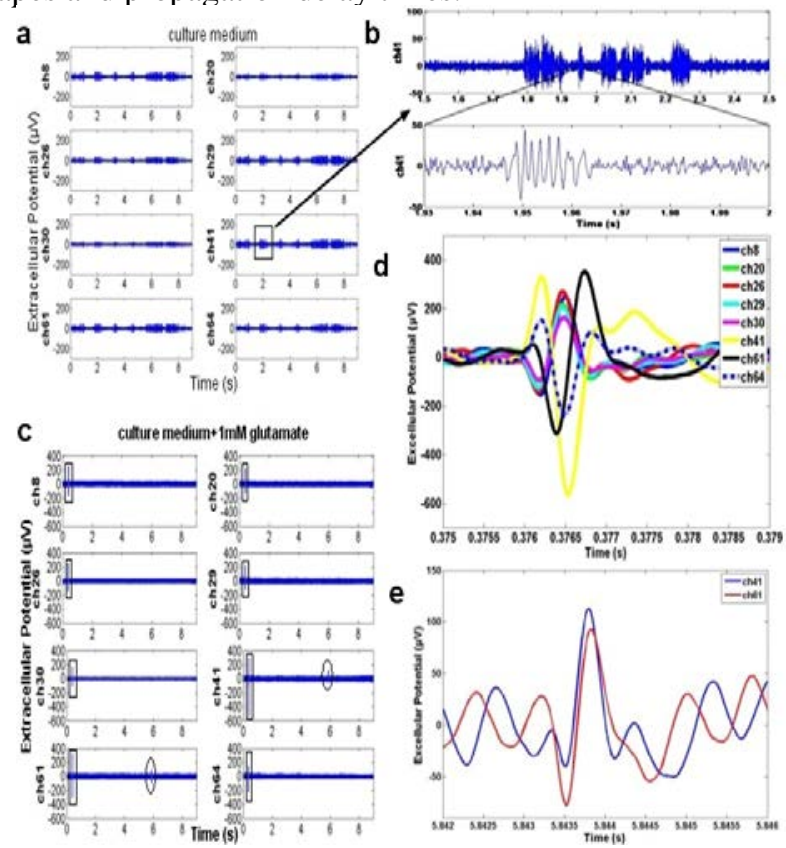

Figure 7. The electrical signals recorded from the patterned OB neurons

(a) The extracellular signals recorded from eight channels in Fig. 6c without the administration of glutamate. (b) The signal marked with a rectangle in Fig. 7a is displayed in detail. (c) The extracellular potential recorded when the patterned neuronswere subjected to $1 \mathrm{mM}$ glutamate. (d)

The spike signals marked with rectangles in Fig. 7c are enlarged and displayed in one picture to observe the phase delay between different signals. (e) Spike signals marked with ellipses in Fig. 7c are enlarged. 


\section{B. Discussion}

The bovine serum albumin can inhibit cell attachment. Albumin from the culture medium rapidly adsorbed to the top surface of the PDMS culture device (hydrophobic) and discouraged cell growth. This prevented cells from adhering to the PDMS surface outside the micro wells and micro trenches. The PLL adsorbed on the walls of micro wells and micro trenches (hydrophilic) facilitated OB cell anchoring.

In current study, the neurons in the micro wells grew more slowly than the neurons on a Petri dish. OB neurons often clung to the corner of the micro wells and grew into irregular shapes, and more than one neuron fell into a micro well. Additionally, some micro trenches were blocked by small cells. These might result from the inappropriate design of the micro wells and micro trenches. Round micro wells were employed for cell patterning by other researchers [14]. This was helpful to keep the regular shape of neurons. Round micro wells and the micro trenches with smaller width should be employed for the further research.

The Extracellular electric field decreases with distance from the cell. Cohen and Miles [13] indicated that the extracellular signal could be obtained from a microelectrode with the maximum distance of $80 \mu \mathrm{m}$ away from the neuron. They constructed a curve relating the peak to peak amplitude of the averaged extracellular signal to the distance between the cell soma and electrode. The curve implied an inverse square relation between the spike amplitude and distance. The depth of micro wells was a key factor affecting the cell-electrode distance. It strongly influenced the signal to noise ratio. When the PDMS culture device was placed upside down over the electrodes, the gap between the cell soma and the electrode was about 20 to $40 \mu \mathrm{m}$. The micro wells with different depths have been used by different researchers $[10,15]$. There is little possibility that the $\mathrm{OB}$ neurons will escape from the micro wells deeper than $20 \mu \mathrm{m}$. Shallower micro wells will decrease the gaps between neurons and the electrodes, which will be benefit for the promotion of signal to noise ratio.

Another method of decreasing the cell-electrode distance may be transferring neurons in the PDMS device to the MEA surface as follows: firstly the neurons will be cultured for several days to be pre-fixed in the PDMS microstructure before their maturation, after the preliminary forming of topological connections, the device will be placed upside down on the MEA surface plated with cell adhesive material such as collagen and fibronectin to guide the neuron adhering. After another cultivation for several days, it may be possible to transfer the neurons from micro wells to the electrode surface. The time of cultivation for the cell prefixing needs to be studied. By means of this method, the neurons in the micro wells may migrate to the surface of MEA while the topological connection between adjacent neurons will be maintained.

The signals from different channels have different shapes (Fig. 7d). Grattarola et al. [16] found the signal shape could be interpreted in terms of time derivatives of the action potential. The order of time derivative was influenced by the cell-electrode coupling. Bove et al. [12] simulated the signal corresponding to an action potential. By varying the cell-electrode coupling and other biophysical parameters, they got signals of different durations, intensities and shapes. Spike shape was also influenced by both of cell geometry and cell size [17].

In the present study, the drug stimulation had a drawback of low spatial resolution because of the uncontrollable diffusion of the glutamate. Micro channels for precise drug delivery or new stimulation methods such as photochemical stimulation should be adopted in the further research.

\section{CONCLUSIONS}

In present work, a simple approach to form an OB neuron network in vitro for the extracellular potential recording was introduced. This was a first attempt to culture the OB neurons in a confined geometry and to place it onto the MEA biochip for the potential recording. However, the unsuitable dimension of the micro wells hindered the growth of $\mathrm{OB}$ neurons into the normal shape and resulted in a low signal to noise ratio of the signal. The microstructures with appropriate sizes and shapes should be designed in the future research. Besides, microfluidic channels for delivering different chemicals to different locations will be added to the current culture device to promote the stimuli resolution and increase the survival time of the neurons. The technique can be used for the research of the OB neuronal network and olfactory biosensors.

\section{ACKNOWLEDGMENT}

This work was financially supported by the National Key Technology R\&D Program of China (no. 2012BAI16B02) and the National Natural Science Foundation of China (nos 81171416, 60875078).

\section{REFERENCES}

[1] N. Uchida, C. Poo and R. Haddad, "Coding and transformations in the olfactory system," Neuroscience, 37(37): 363-385, 2014.

[2] G. M. Shepherd, "The olfactory bulb: a simple system in the mammalian brain," in Handbook of Physiology, Section 1, Vol. 1, Part 2, J. M. Brookhart, V. B. Mountcastle, and E. R. Kandel, Eds. Bethesda: American Physiological Society, 2011, pp. 945-968.

[3] D. H. Coelho and R. M. Costanzo, "Spatial mapping in the rat olfactory bulb by odor and direct electrical stimulation," Otolaryngol.--Head Neck Surg., 155, 2016.

[4] R. Todd Pressler, P. A. Rozman and B. W. Strowbridge, "Voltagedependent intrinsic bursting in olfactory bulb Golgi cells," Learn. Mem., 20: 459-466, 2013.

[5] R. C. Gerkin, S. J. Tripathy and N. N. Urban, "Origins of correlated spiking in the mammalian olfactory bulb,” PNAS, 110: 17083-17088, 2013.

[6] T. Datta-Chaudhuri, R. C. Araneda, P. Abshire and E. Smela, "Olfaction on a chip," Sens. Actuators, B, 235: 74-78, 2016.

[7] D. J. Collins, B. Morahan, J. Garcia-Bustos, C. Doerig, M. Plebanski and A. Neild, "Two-dimensional single-cell patterning with one cell per well driven by surface acoustic waves," Nat. Commun., 6, 2015.

[8] C. J. Pan, H. Qin, Y. D. Nie and H. Y. Ding, "Control of osteoblast cells adhesion and spreading by microcontact printing of extracellular matrix protein patterns," Colloids Surf., B, 104.104C: 18-26, 2013. 
[9] E. Claverol-Tinture, J. Cabestany and X. Rosell, "Multisite recording of extracellular potentials produced by microchannel-confined neurons in-vitro,” IEEE Trans. Biomed. Eng., 54: 331-335, 2007.

[10] M. Bani-Yaghoub, R. Tremblay, R. Voicu, G. Mealing, R. Monette, C. Py, K. Faid, M. Sikorska, "Neurogenesis and neuronal communication on micropatterned neurochips", Biotechnol. Bioeng., 92: 336-345, 2005.

[11] A. Kaneko and Y. Sankai, "Long-term culture of rat hippocampal neurons at low density in serum-free medium: combination of the sandwich culture technique with the three-dimensional nanofibrous hydrogel PuraMatrix," Plos One, 9(7): e102703, 2014.

[12] M. Bove, M. Grattarola, S. Martinoia, G. Verreschi, "Interfacing cultured neurons to planar substrate microelectrodes: characterization of the neuron-to-microelectrode junction,” Bioelectrochem. Bioenerg., 38: 255-265,1995.
[13] I. Cohen and R. Miles, "Contributions of intrinsic and synaptic activities to the generation of neuronal discharges in in vitro hippocampus,” J. Physiol., 524.2: 485-502, 2000.

[14] L. Wang, S. K. Murthy, W. H. Fowle, G. A. Barabino and R. L. Carrier, "Influence of micro-well biomimetic topography on intestinal epithelial Caco-2 cell phenotype," Biomaterials, 30(36): 6825-6834, 2009.

[15] L. Lin, M. Jie, F. Chen, J. Zhang, Z. He and J. Lin, "Efficient cell capture in an agarose-PDMS hybrid chip for shaped 2D culture under temozolomide stimulation," RSC Adv., 6, 2016.

[16] M. Grattarola and S. Martinoia, "Modeling the neuronmicrotransducer junction: from extracellular to patch recording," IEEE Trans. Biomed. Eng., 40: 35-41,1993.

[17] T. V. Ness, C. Chintaluri, J. Potworowski, S. Leski, H. Glabska and G. T. Einevoll, "Modelling and analysis of electrical potentials recorded in microelectrode arrays (MEAs)," Neuroinformatics, 13(4):1-24. 